\title{
Pretargeted Versus Directly Targeted Radioimmunotherapy Combined with Anti-CD20 Antibody Consolidation Therapy of Non-Hodgkin Lymphoma
}

Robert M. Sharkey ${ }^{1}$, Habibe Karacay ${ }^{1}$, Christine R. Johnson ${ }^{1}$, Samuel Litwin ${ }^{2}$, Edmund A. Rossi ${ }^{3}$, William J. McBride ${ }^{4}$, Chien-Hsing Chang ${ }^{3,4}$, and David M. Goldenberg ${ }^{1}$

${ }^{I}$ Garden State Cancer Center at the Center for Molecular Medicine and Immunology, Belleville, New Jersey; ${ }^{2}$ Fox Chase Cancer Center, Philadelphia, Pennsylvania; ${ }^{3}$ IBC Pharmaceuticals, Inc., Morris Plains, New Jersey; and ${ }^{4}$ Immunomedics, Inc., Morris

Plains, New Jersey

\begin{abstract}
We determined whether therapeutic responses using a bispecific antibody that pretargeted ${ }^{90} \mathrm{Y}$-hapten-peptide radioimmunotherapy or a directly radiolabeled, humanized, ${ }^{90}$ Y-anti-CD20 IgG (veltuzumab) could be improved by combining these treatments with unlabeled humanized antibodies against CD22 (epratuzumab), CD74 (milatuzumab), or veltuzumab. Methods: Nude mice bearing established subcutaneous Ramos human Burkitt lymphoma were treated with antibodies alone or in combination with pretargeted radioimmunotherapy (PT-RAIT) or radioimmunotherapy, and tumor growth was monitored. Biodistribution studies examined the effect that predosing with unlabeled veltuzumab had on radioimmunotherapy and PT-RAIT targeting. Results: None of the unconjugated antibodies was effective against established and rapidly growing xenografts, but PT-RAIT, at approximately $30 \%$ of its maximum tolerated dose, and radioimmunotherapy alone, at its maximum tolerated dose, were able to arrest growth and even entirely ablate tumors in some animals. Only combinations with veltuzumab improved therapeutic responses, most significantly when a veltuzumab regimen (weekly, $1.0 \mathrm{mg}$ followed by $3 \times 0.5 \mathrm{mg}$ ) was initiated $1 \mathrm{wk}$ after PT-RAIT or ${ }^{90} Y$-veltuzumab. Biodistribution data indicated that when unlabeled veltuzumab ( 1.0 or $0.25 \mathrm{mg}$ ) was administered in advance of the radiolabeled veltuzumab or bispecific antibody injection, tumor uptake was significantly reduced $\left({ }^{111}\right.$ In-veltuzumab, $47 \%$ and $25 \%$, respectively; ${ }^{111}$ In-hapten-peptide, $74 \%$ and $49 \%$, respectively). Despite an approximately $50 \%$ decrease in radioactivity uptake in the tumor, antitumor responses were not diminished significantly for ${ }^{90} \mathrm{Y}$-veltuzumab, and in the case of PT-RAIT responses were improved. However, higher amounts of predosed veltuzumab reduced the effects of PT-RAIT. Conclusion: These studies suggest that administering unlabeled anti-CD20 lgG therapy after the radioactivity dose provides the best efficacy and that the amount of unlabeled anti-CD20 IgG administered as a predose to anti-CD20-targeted radionuclide therapy should be minimized.
\end{abstract}

Received Sep. 28, 2008; revision accepted Dec. 2, 2008.

For correspondence or reprints contact: Robert M. Sharkey, Garden State Cancer Center at the Center for Molecular Medicine and Immunology, 520 Belleville Ave., Belleville, NJ 07109.

E-mail: rmsharkey@gscancer.org

COPYRIGHT $\odot 2009$ by the Society of Nuclear Medicine, Inc.
Key Words: non-Hodgkin lymphoma; bispecific antibody; pretargeting; radioimmunotherapy; veltuzumab; ${ }^{90} \mathrm{Y}$

J Nucl Med 2009; 50:444-453

DOI: 10.2967/jnumed.108.058602

A nti-CD20 antibodies are exceptionally good therapeutics for non-Hodgkin lymphoma (NHL), with both unlabeled and radiolabeled antibodies approved or being evaluated for frontline use (1-5). We and others have focused on pretargeted methods for delivery of radionuclides, because these treatments are less toxic and more effective than is direct radioimmunotherapy in animal models (6-10). Pretargeted radioimmunotherapy (PT-RAIT) separates the antibody-targeting step from the delivery of the radionuclide. After time has been allowed for pretargeting of a bispecific antibody (bsMAb), the radionuclide coupled to a small compound is given, instantly permeates the blood vessels to localize rapidly and selectively in pretargeted tumors, and just as quickly is removed by urinary excretion, leaving only trace amounts of the radionuclide in the body (7). The rapid clearance from blood and tissues reduces radiation exposure, and hence the extent and duration of hematologic toxicity is markedly reduced. Tumor uptake of this small, pretargeted compound can achieve levels similar to that of the directly radiolabeled antibody, resulting in a more intense radiation dose rate and a higher overall radiation-absorbed dose in the tumor at equitoxic levels $(10,11)$.

In contrast to radioimmunotherapy, which exerts antitumor activity from radiation and from the effector or apoptotic signaling functions contributed by the large amount of unlabeled anti-CD20 IgG administered before the radioimmunoconjugate $(\sim 450 \mathrm{mg} /$ dose $\times 2$ doses $)$, the newer forms of recombinant proteins used for pretargeting lack the 
Fc (crystallizable fragment) portion of an antibody that contributes to the antitumor activity of the antibody itself $(12,13)$. Although in vitro studies have shown that the divalent hapten-peptide can cross-link an anti-CD20 $\times$ antihapten bsMAb and enhance apoptosis, in vivo studies in nude mice bearing established tumors, versus untreated animals, indicated no significant therapeutic improvement of the bsMAb with the unlabeled hapten-peptide $(9,14)$. Therefore, the antitumor activity elicited with PT-RAIT is associated solely with the radiation.

We hypothesized that the addition of an anti-CD20 IgG might enhance the therapeutic effect of anti-CD20 bsMAb PT-RAIT. Indeed, in NHL and multiple myeloma animal models, several unconjugated antibodies have therapeutic activity (15-18), and we and others have described enhanced antitumor responses when combining anti-CD20 IgG with radioimmunoconjugates binding to other antigens (19-21). Thus, a logical question is whether the therapeutic activity of PT-RAIT could be enhanced by combining it with unconjugated antibodies. Herein, we examine the addition of unlabeled humanized anti-CD22 (epratuzumab; Immunomedics, Inc.), anti-CD74 (milatuzumab; Immunomedics, Inc.), and anti-CD20 IgG (veltuzumab; Immunomedics, Inc.) to our standard anti-CD20-bsMAb pretargeting procedure (9) and to ${ }^{90}$ Y-veltuzumab radioimmunotherapy. Only the antiCD20 IgG enhanced the therapeutic activity of the pretargeting procedure or of a directly radiolabeled anti-CD20 IgG, and only when administered after the radioconjugate had localized in the tumor. These results have important implications for the future design of clinical studies with this pretargeting procedure in NHL and potentially also for other current radioimmunotherapy methods.

\section{MATERIALS AND METHODS}

\section{Antibodies and Peptides}

Veltuzumab (hA20 or Immu-106), a humanized anti-CD20 IgG, and its 1,4,7,10-tetraazacyclododecane- $N, N^{\prime}, N^{\prime \prime}, N^{\prime \prime \prime}$-tetraacetic acid (DOTA) conjugate, were provided by Immunomedics, Inc. In vitro and in vivo activity of veltuzumab against the Burkitt lymphoma cell line (B-cell) was reported previously $(16,18)$. The trivalent-Fab bsMAb TF4 (157 kDa), which has divalent binding to CD20 and monovalent binding to the hapten histamine-succinylglycine (HSG), and the di-HSG-DOTA-peptide IMP-288 were provided by IBC Pharmaceuticals, Inc. and Immunomedics, Inc., respectively, and were described previously (9). Epratuzumab (hLL2, humanized anti-CD22 IgG) and milatuzumab (hLL1, humanized anti-CD74 IgG) also were provided by Immunomedics, Inc. The antitumor activity of these antibodies in animal models has been studied (15-17). The antitumor activity of epratuzumab and veltuzumab in NHL patients has been reported $(22,23)$; phase I clinical testing with milatuzumab in NHL, chronic lymphocytic leukemia, and multiple myeloma is under way.

${ }^{111} \mathrm{In}$ - and ${ }^{90} \mathrm{Y}$-IMP-288 and ${ }^{111} \mathrm{In}$ - and ${ }^{90} \mathrm{Y}$-veltuzumab were prepared as reported previously $(9,24)$. Quality-control testing showed greater than or equal to $97 \%$ of the radioactivity bound to the test articles, with greater than $95 \%$ retention of immunoreactivity. Immunoreactivity of radiolabeled veltuzumab was deter- mined by size-exclusion high-pressure liquid chromatography (SE-HPLC) after mixing with an antiidiotype antibody (9). When radiolabeled IMP-288 was mixed with excess TF4, the peptide shifted to a higher molecular weight fraction by SE-HPLC. The subsequent addition of the antiveltuzumab idiotype antibody resulted in a further quantitative shift in molecular size, illustrating the bispecific binding of TF4.

\section{In Vivo Studies}

All studies were performed in accordance with protocols approved by the Center for Molecular Medicine and Immunology's Institutional Animal Care and Use Committee. The Ramos human Burkitt lymphoma cell line was purchased from the American Tissue Culture Collection. On the day of implantation, $1 \times 10^{7}$ cells $(0.25 \mathrm{~mL}$; viability, $>95 \%)$ were injected subcutaneously in 5- to 6-wk-old female BALB/c nude mice purchased from the National Cancer Institute's Charles River Facility. Within $17 \mathrm{~d}$, tumors of palpable size and progressive growth were observed. Tumors were then measured by caliper in 3 dimensions, with the size reported as the product of these measurements $\left(\mathrm{cm}^{3}\right)$.

\section{Biodistribution Studies}

Animals bearing established tumors were selected and given intravenous injections of the test articles at the doses and schedules indicated. For pretargeting, TF4 was always administered $29.5 \mathrm{~h}$ in advance of ${ }^{111}$ In-IMP-288 and administered at a 25 -fold excess of the moles of IMP-288 administered. Biodistribution studies were performed with the same amounts of TF4 and IMP288 as were used in the therapy studies, with $355 \mu \mathrm{g}(\sim 2.25$ $\mathrm{nmol})$ of TF4 and $0.09 \mathrm{nmol}$ of IMP-288 (1.11 MBq [30 $\mu \mathrm{Ci}])$ or $50 \mu \mathrm{g}$ of ${ }^{111}$ In-veltuzumab (0.74 MBq [20 $\left.\left.\mu \mathrm{Ci}\right]\right)$. In some studies, a predose of 0.25 or $1.0 \mathrm{mg}$ of veltuzumab was administered either $1 \mathrm{~d}$ or $1 \mathrm{~h}$ in advance of the TF4 or ${ }^{111}$ In-veltuzumab injection, respectively. The $0.25-$ or $1.0-\mathrm{mg}$ amount of veltuzumab is equivalent to a human dose of about 1 or $4 \mathrm{mg} / \mathrm{kg}$, respectively, based on a conversion factor using the following formula: $\mathrm{mg} / \mathrm{kg}$ dose in a 20-g mouse divided by 12.3 equals a human equivalent $\mathrm{mg} / \mathrm{kg}$ dose (25). At the times indicated, groups of animals were anesthetized, bled by cardiac puncture, and then euthanized by cervical dislocation. Tumors and tissues were weighed, and ${ }^{111} \mathrm{In}-$ activity was determined by $\gamma$-scintillation. A counting standard was measured at the same time and used to convert counts per minute per gram of tissue to percentage injected dose per gram $(\% \mathrm{ID} / \mathrm{g})$.

\section{Therapy Studies}

When tumors were greater than or equal to $0.4 \mathrm{~cm}^{3}$, animals were sorted into groups with a similar tumor-size distribution. They were monitored daily, with tumor and body weights measured at least twice weekly. All articles were administered by tailvein injection in a volume of no more than $0.2 \mathrm{~mL}$. Unlabeled veltuzumab, epratuzumab, and milatuzumab treatment regimens consisted of 4 weekly injections, starting with 1-2 mg (day 0) and then $3 \times 0.5$-mg injections. For PT-RAIT, TF4 was administered on day -1 , with $7.4-9.25 \mathrm{MBq}(0.20-0.25 \mathrm{mCi})$ of ${ }^{90} \mathrm{Y}-\mathrm{IMP}-288$ administered $29.5 \mathrm{~h}$ later (day 0 ). This level of radioactivity represents no more than $30 \%$ the maximum tolerated dose (MTD), and although therapeutically active, this level was not curative in all animals (9). For radioimmunotherapy, $4.81 \mathrm{MBq}(0.13 \mathrm{mCi})$ or less of ${ }^{90} \mathrm{Y}$-veltuzumab $(50 \mu \mathrm{g})$ were administered (day 0$)$. This dose was considered the MTD because prior studies indicated that $5.55 \mathrm{MBq}(0.15 \mathrm{mCi})$ resulted in at least a $90 \%$ reduction in total 
white blood cells for more than $1 \mathrm{wk}$, and treatment-related toxicity (TRT) occurred (9).

Consolidation therapy with veltuzumab consisted of the same unlabeled veltuzumab treatment regimen mentioned above but initiated $1 \mathrm{wk}$ after PT-RAIT or radioimmunotherapy. In predosing studies, veltuzumab was administered $1 \mathrm{~d}$ in advance of the TF4 (day -2) or ${ }^{90}$ Y-veltuzumab (day -1 ) injections. Unlabeled epratuzumab was administered $2 \mathrm{~d}$ (day 2) after 7.4 MBq of PTRAIT, and the unlabeled milatuzumab treatment regimen was given the same day as $9.25 \mathrm{MBq}$ of PT-RAIT. Another study combined the unlabeled epratuzumab treatment regimen with ${ }^{90}$ Y-veltuzumab, starting on day 0.

No more than 5 animals were housed per cage, and bedding was changed within a few hours of PT-RAIT, to remove excess excreted ${ }^{90}$ Y-activity. Animals were removed from the study if the tumor size exceeded $2.5 \mathrm{~cm}^{3}$, if the body weight decreased by more than $20 \%$ of the starting weight, or if there were any other signs of morbidity.

\section{Statistical Considerations}

Biodistribution data were compared using a 2-tailed $t$ test (GraphPad Prism, version 4.0; GraphPad Software). Therapeutic efficacy was based on Kaplan-Meier survival analysis (GraphPad Prism) using a log-rank test, in which survival was based on the approximate time required for tumor progression to $2.5 \mathrm{~cm}^{3}$ (differences based on 2-tailed $P$ values, requiring $P<0.05$ ). Mice that failed to reach this endpoint (most were tumor-free) were censored at the end of the study. Animals withdrawn because of TRT or nontreatment-related toxicity (NTRT) were censored at the time of their removal. A few tumors progressed and then regressed well after the time that such regression could be attributed with certainty to treatment and were considered as treatment failures at the time of their maximum size. The fraction of animals cured (S) was based on those with no evidence of disease at the end of study divided by the number of animals in the group that could be assessed unambiguously (i.e., animals that were censored before the study end were subtracted from the study total).

\section{RESULTS}

Figure 1 summarizes the therapeutic activity of the unlabeled antibodies, PT-RAIT alone, and several combinations based on time required for tumors to progress to $2.5 \mathrm{~cm}^{3}$ (i.e., survival). The individual growth curves, with median survival times, are shown in Supplemental Figure 1 (supplemental materials are available online only at http://jnm.snmjournals. org). Untreated animals progressed from an average starting size of $0.71 \pm 0.24 \mathrm{~cm}^{3}$ to $2.5 \mathrm{~cm}^{3}$ or greater within $1 \mathrm{wk}$. None of the unconjugated antibodies controlled tumor growth significantly. All animals that were administered 9.25 MBq of PT-RAIT alone experienced a complete response (no tumor visible); within a few weeks, however, 7 of 10 tumors progressed, yielding a median survival of $4.9 \mathrm{wk}$ (vs. untreated, $P<0.0001)$. Three animals were tumor-free at $16 \mathrm{wk}$. Animals treated with 7.4 MBq of PT-RAIT combined with epratuzumab also showed an initial response, but 9 of 10 animals progressed quickly, with 1 animal having complete tumor ablation. The median survival time in this combination group was shorter than the previously mentioned PT-RAIT-alone group (2.8 vs. 4.9 wk), because a lower dose of the ${ }^{90}$ Y-IMP-288 was

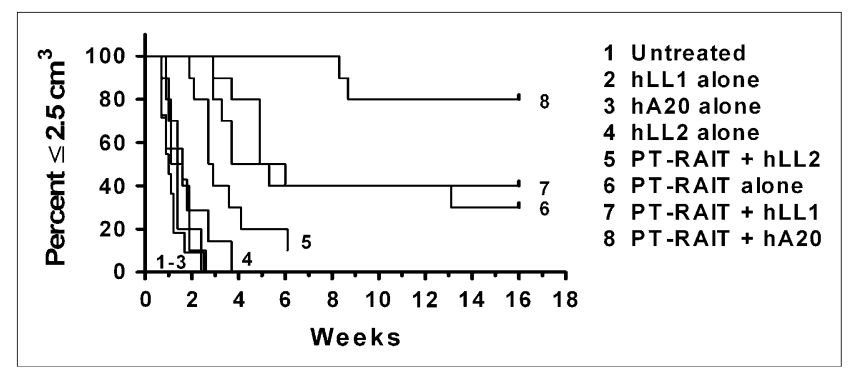

FIGURE 1. Survival curves for PT-RAIT combined with unconjugated antibodies with PT-RAIT. BALB/c nude mice bearing subcutaneous Ramos tumors underwent PT-RAIT with or without unconjugated antibody. Tumor size was monitored for $16 \mathrm{wk}$. Animals were removed from study when tumor progressed to greater than or equal to $2.5 \mathrm{~cm}$. Growth curves for individual groups and animals are shown in Supplemental Figure 1 (hLL1, milatuzumab; hA20, veltuzumab; hLL2, epratuzumab).

administered (7.4 vs. 9.25 MBq). The milatuzumab plus PT-RAIT survival time was no better than the PT-RAIT-alone survival time $(P=0.5540)$. However, when a veltuzumab treatment regimen was initiated $1 \mathrm{wk}$ after PT-RAIT, complete responses were induced in all animals for at least $4 \mathrm{wk}$; after 16 wk, 8 of 10 animals were tumor-free, providing a significantly improved response, compared with the untreated $(P<0.0001)$ and all other treatment groups.

If the veltuzumab regimen was started so that the initial 1-mg dose was administered $1 \mathrm{~d}$ in advance of the TF4 injection to simulate a predose commonly given to reduce splenic targeting of the directly radiolabeled anti-CD20 IgG, the antitumor effect was impaired significantly, compared with the PT-RAIT-alone group included in this set of animals (Fig. 2). The antitumor effect was also impaired significantly with this regimen, compared with the earlier study, for which veltuzumab was initiated $1 \mathrm{wk}$ after ${ }^{90}$ Y-IMP-288 had been administered (Fig. 1). In this study, although all animals in the PT-RAIT-alone group initially had a complete response, none of the tumors in the veltuzumab-plus-PT-RAIT group regressed to smaller than their starting size before progressing and the median time to $2.5 \mathrm{~cm}^{3}$ was reduced significantly (2.2 and $4.4 \mathrm{wk}$ for veltuzumab plus PT-RAIT and PT-RAIT alone, respectively; $P=0.0013)$. Thus, antitumor responses were inhibited significantly by shifting the veltuzumab treatment regimen so that a single human-equivalent dose of approximately $4 \mathrm{mg}$ of veltuzumab per kilogram was administered before PT-RAIT.

These data suggested that the predose reduced tumor uptake of the ${ }^{90} \mathrm{Y}$-IMP-288. Biodistribution data showed that tumor uptake $24 \mathrm{~h}$ after injection of the ${ }^{111}$ In-IMP-288 was decreased by $74 \%$ when $1 \mathrm{mg}$ of veltuzumab was administered $1 \mathrm{~d}$ in advance of the TF4 bsMAb injection, compared with when no predose was administered $(2.96 \pm$ $0.75 \% \mathrm{ID} / \mathrm{g}$ from $11.28 \pm 0.21 \% \mathrm{ID} / \mathrm{g} ; P<0.0001$ ) (Table 1 , set A). However, the concentration of ${ }^{111}$ In-IMP-288 in 


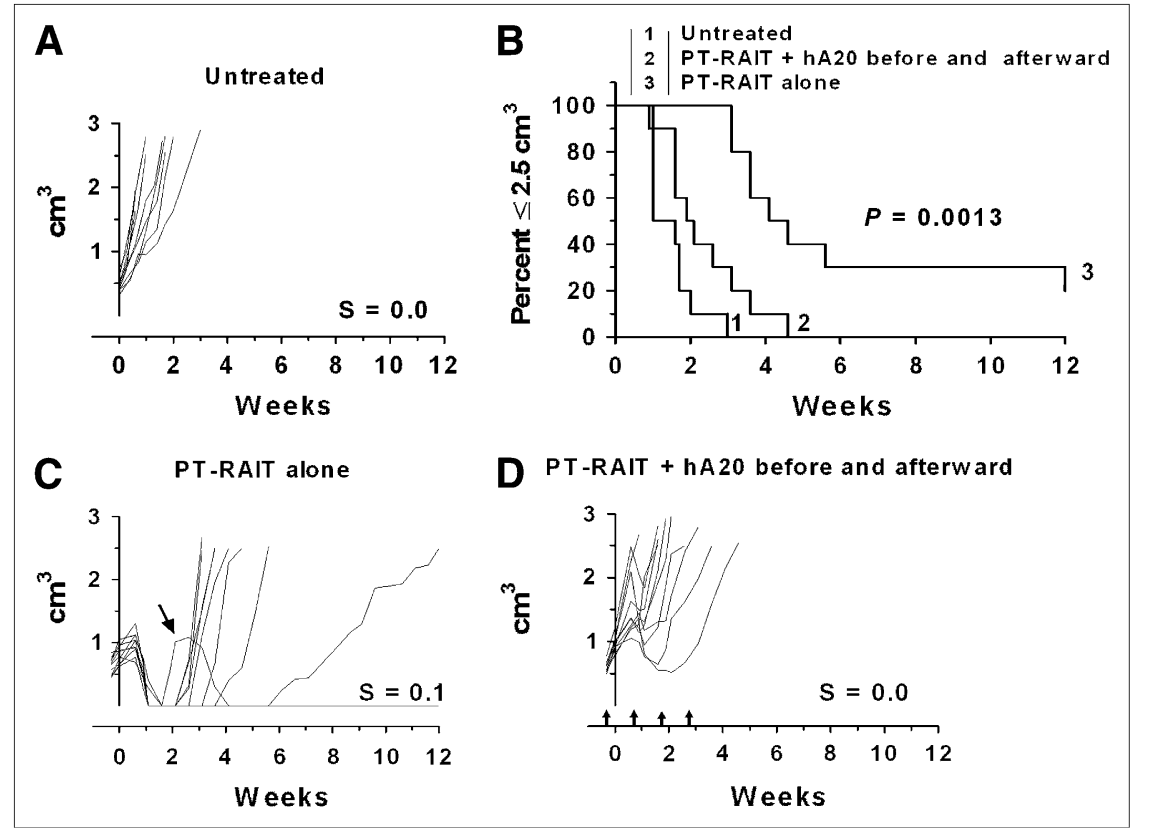

FIGURE 2. Combining PT-RAIT with veltuzumab treatment regimen starting $1 \mathrm{~d}$ in advance of TF4 injection: Untreated $(A)$, percentage of animals remaining with tumors less than or equal to $2.5 \mathrm{~cm}^{3}$ (B), PT-RAIT alone $(2.25$ $\mathrm{nmol} / 355 \mu \mathrm{g}$ of TF4, followed $29 \mathrm{~h}$ later with $9.25 \mathrm{MBq} / 0.09 \mathrm{nmol}$ of ${ }^{90} \mathrm{Y}-\mathrm{IMP}$ 288) (C), and PT-RAIT plus hA20 before and after PT-RAIT (same as treatment $\mathrm{C}$, but $1 \mathrm{mg}$ of veltuzumab was administered $1 \mathrm{~d}$ before TF4 and then followed by 3 weekly injections of $0.5 \mathrm{mg}$ of veltuzumab) (D). $\mathrm{T}_{0}$ is time ${ }^{90} \mathrm{Y}-\mathrm{IMP}$ 288 was administered. Arrows show times when veltuzumab was administered.

the blood was unchanged. Additional studies examined the effect of lowering the predose to $0.25 \mathrm{mg}$, which is equivalent to a $1 \mathrm{mg} / \mathrm{kg}$ dose in humans, a level that was found to improve biodistribution of ${ }^{90} \mathrm{Y}$-ibritumomab tiuxetan (26). Under these conditions, tumor uptake measured $24 \mathrm{~h}$ after the ${ }^{111} \mathrm{In}-\mathrm{IMP}-288$ injection was reduced by $48.5 \%(13.40 \pm 0.60 \% \mathrm{ID} / \mathrm{g}$ vs. $6.82 \pm 0.75 \% \mathrm{ID} / \mathrm{g})$, with no effect on blood or tissue concentrations (Table 1, set B). Interestingly, when veltuzumab was administered just $1 \mathrm{~h}$ in advance of TF4, tumor targeting and biodistribution were similar to that found with the 1-d predose of either a 1.0- or a 0.25 -mg predose (Table 1 , set $\mathrm{C}$ ).

These results led to another therapy study in which animals were administered the veltuzumab therapy regimen starting with just $0.25 \mathrm{mg}$ of veltuzumab $1 \mathrm{~d}$ before PTRAIT, followed by weekly $0.5-\mathrm{mg}$ doses. In this setting, the median time to $2.5 \mathrm{~cm}^{3}$ for the PT-RAIT-alone group of 4.4 wk was significantly shorter than that for the predosed PTRAIT group (9.6 wk; $P=0.0389$ ) (Fig. 3). Thus, even with a reduction in tumor uptake of approximately $50 \%$, this

\section{TABLE 1. Effect of Veltuzumab Advanced Dose on Biodistribution of ${ }^{111} \mathrm{In}$-Veltuzumab IgG or TF4-Pretargeted} ${ }^{111}$ In-IMP-288

\begin{tabular}{|c|c|c|c|c|c|c|c|}
\hline \multirow[b]{2}{*}{ Antibody and target } & \multicolumn{2}{|c|}{ Set A } & \multicolumn{2}{|c|}{ Set B } & \multicolumn{3}{|c|}{ Set C } \\
\hline & No predose & $1.0 \mathrm{mg} / 1 \mathrm{~d}$ & No predose & $0.25 \mathrm{mg} / 1 \mathrm{~d}$ & No predose & $0.25 \mathrm{mg} / 1 \mathrm{~h}$ & $1.0 \mathrm{mg} / 1 \mathrm{~h}$ \\
\hline \multicolumn{8}{|l|}{${ }^{111}$ In-veltuzumab IgG } \\
\hline Tumor & $16.9 \pm 4.1$ & $9.0 \pm 1.4$ & $22.5 \pm 3.8$ & $16.8 \pm 2.0$ & $22.1 \pm 2.7$ & $18.1 \pm 2.5$ & $13.2 \pm 4.5$ \\
\hline Tumor weight & $0.532 \pm 0.124$ & $0.677 \pm 0.302$ & $0.264 \pm 0.081$ & $0.255 \pm 0.047$ & $0.307 \pm 0.150$ & $0.323 \pm 0.144$ & $0.352 \pm 0.258$ \\
\hline Liver & $3.5 \pm 0.6$ & $4.0 \pm 0.5$ & $4.7 \pm 1.7$ & $5.6 \pm 0.4$ & $4.1 \pm 0.4$ & $4.7 \pm 1.3$ & $4.7 \pm 0.3$ \\
\hline Spleen & $4.3 \pm 1.8$ & $4.3 \pm 0.5$ & $5.4 \pm 4.0$ & $5.6 \pm 0.8$ & $3.6 \pm 0.8$ & $4.1 \pm 1.0$ & $4.3 \pm 0.5$ \\
\hline Kidneys & $5.0 \pm 0.7$ & $7.1 \pm 1.0$ & $4.8 \pm 0.5$ & $5.40 \pm 0.8$ & $5.2 \pm 0.6$ & $6.7 \pm 1.2$ & $6.6 \pm 0.3$ \\
\hline Lungs & $6.9 \pm 1.5$ & $7.8 \pm 1.0$ & $5.9 \pm 1.0$ & $7.2 \pm 1.5$ & $6.0 \pm 2.4$ & $6.0 \pm 1.0$ & $7.7 \pm 2.9$ \\
\hline Blood & $14.8 \pm 2.0$ & $20.3 \pm 2.6$ & $16.1 \pm 2.3$ & $22.6 \pm 2.3$ & $15.2 \pm 1.9$ & $18.2 \pm 2.8$ & $19.8 \pm 2.2$ \\
\hline \multicolumn{8}{|l|}{$\begin{array}{l}\text { TF4-pretargeted } \\
111 \text { In-IMP-288 }\end{array}$} \\
\hline Tumor & $11.28 \pm 0.21^{*}$ & $2.96 \pm 0.75^{\star}$ & $13.40 \pm 0.60$ & $6.82 \pm 0.75^{\star}$ & $13.23 \pm 1.81$ & $6.74 \pm 1.51$ & $2.40 \pm 0.61$ \\
\hline Tumor weight & $0.559 \pm 0.209$ & $0.754 \pm 0.253$ & $0.303 \pm 0.061$ & $0.469 \pm 0.180$ & $0.228 \pm 0.067$ & $0.329 \pm 0.131$ & $0.195 \pm 0.063$ \\
\hline Liver & $0.43 \pm 0.12$ & $0.68 \pm 0.17$ & $0.63 \pm 0.10$ & $0.71 \pm 0.17$ & $0.66 \pm 0.30$ & $0.81 \pm 0.14$ & $0.77 \pm 0.25$ \\
\hline Spleen & $0.63 \pm 0.23$ & $1.38 \pm 0.52$ & $1.40 \pm 0.71$ & $1.40 \pm 0.56$ & $1.32 \pm 0.98$ & $1.29 \pm 0.69$ & $1.23 \pm 0.51$ \\
\hline Kidneys & $1.14 \pm 0.14$ & $1.50 \pm 0.09$ & $1.38 \pm 0.18$ & $1.31 \pm 0.26$ & $1.53 \pm 0.27$ & $1.46 \pm 0.24$ & $1.27 \pm 0.15$ \\
\hline Lungs & $0.37 \pm 0.03$ & $0.68 \pm 0.39$ & $0.34 \pm 0.06$ & $0.42 \pm 0.09$ & $0.48 \pm 0.14$ & $0.38 \pm 0.04$ & $0.50 \pm 0.22$ \\
\hline Blood & $0.01 \pm 0.00$ & $0.02 \pm 0.01$ & $0.02 \pm 0.00$ & $0.02 \pm 0.01$ & $0.02 \pm 0.01$ & $0.02 \pm 0.00$ & $0.02 \pm 0.01$ \\
\hline
\end{tabular}

Animals were necropsied either $3 \mathrm{~d}$ after ${ }^{111}$ In-veltuzumab injection or $24 \mathrm{~h}$ after ${ }^{111} \mathrm{In}$-IMP-288 injection $\left({ }^{111} \mathrm{In}-\mathrm{IMP}-288\right.$ was administered $29.5 \mathrm{~h}$ after TF4). \%ID/g for each groups is shown (mean \pm SD; $n=5 /$ group, except where indicated by *, where $n=4$ ). 
FIGURE 3. Effect of single predose of veltuzumab $(0.25 \mathrm{mg})$ on PT-RAIT: untreated $(A)$, percentage of animals remaining with tumors less than or equal to $2.5 \mathrm{~cm}^{3}$ (B), PT-RAIT alone $(2.25$ $\mathrm{nmol} / 355 \mu \mathrm{g}$ of TF4, followed $29 \mathrm{~h}$ later with $9.25 \mathrm{MBq} / 0.09 \mathrm{nmol}$ of ${ }^{90} \mathrm{Y}$-IMP288) (C), and PT-RAIT plus hA20 before and after PT-RAIT (same as treatment $\mathrm{C}$, but $0.25 \mathrm{mg}$ of veltuzumab [hA20] was administered $1 \mathrm{~d}$ before TF4 [1 animal was removed at $8.7 \mathrm{wk}$ for NTRT]) (D). $T_{0}$ is time ${ }^{90} \mathrm{Y}-\mathrm{IMP}-288$ was administered.

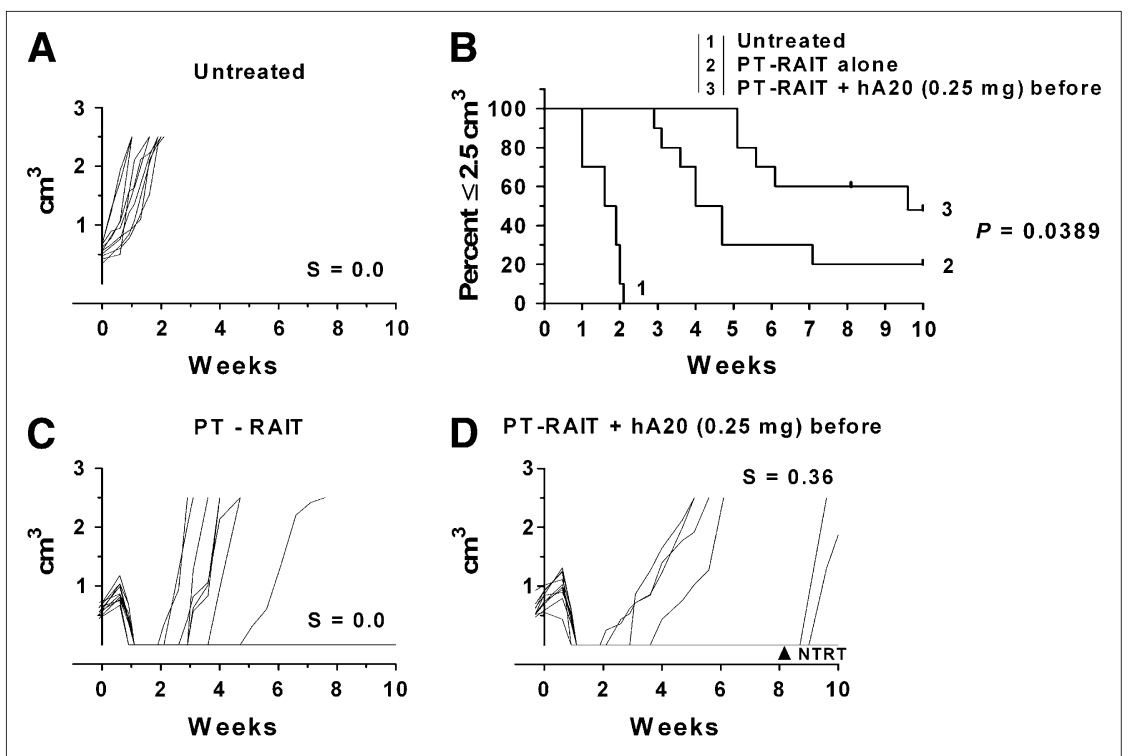

veltuzumab predose with consolidation antibody therapy enhanced the response.

We next asked how direct radioimmunotherapy with ${ }^{90}$ Y-veltuzumab would be affected by combinations with unlabeled antibody therapy. As shown in Figure 4, all tumors responded initially to the ${ }^{90} \mathrm{Y}$-veltuzumab treatment, with 5 partial responses, 5 complete responses, and 1 animal remaining tumor-free for $18 \mathrm{wk}$ (Fig. 4D). One animal was removed from the study because of severe body weight loss at $3.4 \mathrm{wk}$, but this TRT was likely due to the lower body weight of this animal $(16.5 \mathrm{~g})$ than that of the other animals $(19.1 \pm 1.4 \mathrm{~g})$.

Earlier studies had indicated that unlabeled veltuzumab amplified the therapeutic response to ${ }^{90} \mathrm{Y}$-epratuzumab (antiCD22) when the same veltuzumab regimen was initiated $1 \mathrm{~d}$ before the ${ }^{90}$ Y-epratuzumab injection (21). However, a similar treatment regimen of unlabeled epratuzumab added to ${ }^{90} \mathrm{Y}-$ veltuzumab did not affect the therapeutic response, with the median survival time being $3.4 \mathrm{wk}$, compared with $4.4 \mathrm{wk}$ for ${ }^{90}$ Y-veltuzumab alone $(P=0.2281)$ (Fig. 4E). When veltuzumab treatment was initiated $1 \mathrm{~d}$ in advance of ${ }^{90} \mathrm{Y}$ veltuzumab (Fig. 4F), antitumor responses were the same as with ${ }^{90} \mathrm{Y}$-veltuzumab alone, but when the veltuzumab treatment was started 1 wk after ${ }^{90}$ Y-veltuzumab, survival improved significantly $(P=<0.0001)$ (Fig. 4G). Although 6 animals were tumor-free at the end of the study, 1 animal had progressed and regressed on 2 separate occasions. Because the initial progression occurred $6 \mathrm{wk}$ after the last veltuzumab injection, this was considered a treatment failure with progression at week 9.4 , when the tumor was $0.53 \mathrm{~cm}^{3}$. One NTRT caused the removal of an animal at $10.4 \mathrm{wk}$ that had no evidence of tumor at the time.

In this same study, 2 additional groups of animals underwent PT-RAIT for direct comparison with ${ }^{90} \mathrm{Y}$-veltuzumab. Five animals were tumor-free at the end of the study in the PT-RAIT-alone group (Fig. 4B). Tumor progression had occurred in 2 animals at 7 and $12 \mathrm{wk}$ after treatment, but because the tumors regressed without any additional intervention, they were considered treatment failures with progression at these times, yielding a median time to $2.5 \mathrm{~cm}^{3}$ for PT-RAIT alone of $6.8 \mathrm{wk}$, which was not significantly better than with ${ }^{90}$ Y-veltuzumab alone $(P=0.0878)$. When veltuzumab was initiated 1 wk after PT-RAIT, tumor growth was completely arrested in all tumors for at least $12 \mathrm{wk}$, when 2 animals had tumor recurrence (Fig. 4C). Thus, consolidation treatment added to PT-RAIT significantly improved responses over PT-RAIT alone $(P=$ 0.0095), confirming the results of the previous study. On the basis of the log-rank test, the antitumor response was similar when consolidation veltuzumab therapy was added to ${ }^{90}$ Y-veltuzumab or PT-RAIT ( $\left.P=0.1316\right)$, but PT-RAIT was administered at only approximately $30 \%$ its MTD, whereas ${ }^{90}$ Y-veltuzumab was at its MTD. Additionally, when the survival curve for the PT-RAIT combined with veltuzumab that started with the 1-mg predose was compared with the same regimen, but with ${ }^{90} \mathrm{Y}$-veltuzumab, a greater negative impact on PT-RAIT was observed $(P=$ 0.0007).

Biodistribution studies provided insights into the differences in responses between direct and indirect targeted therapy. Animals administered ${ }^{111} \mathrm{In}$-veltuzumab alone had $16.9 \pm 4.1 \% \mathrm{ID} / \mathrm{g}$ in the tumor $3 \mathrm{~d}$ after injection (Table 1 , set A), but animals administered $1 \mathrm{mg}$ of veltuzumab before the radioimmunoconjugate had a $47 \%$ reduction in uptake $(9.0 \pm 1.4 \% \mathrm{ID} / \mathrm{g} ; P=0.003)$. Concentrations of the radioimmunoconjugate in the blood also increased significantly by $37 \%(P=0.005)$, which potentially could exacerbate hematologic toxicity. In a separate study examining the impact of a $0.25-\mathrm{mg}$ predose, tumor uptake $3 \mathrm{~d}$ after ${ }^{111}$ In-veltuzumab decreased $25 \%$, from $22.5 \pm 3.8$ $\% \mathrm{ID} / \mathrm{g}$ to $16.8 \pm 2.0 \% \mathrm{ID} / \mathrm{g}$ when $0.25 \mathrm{mg}$ was administered $1 \mathrm{~d}$ earlier (Table 1, set B). Blood concentrations re- 


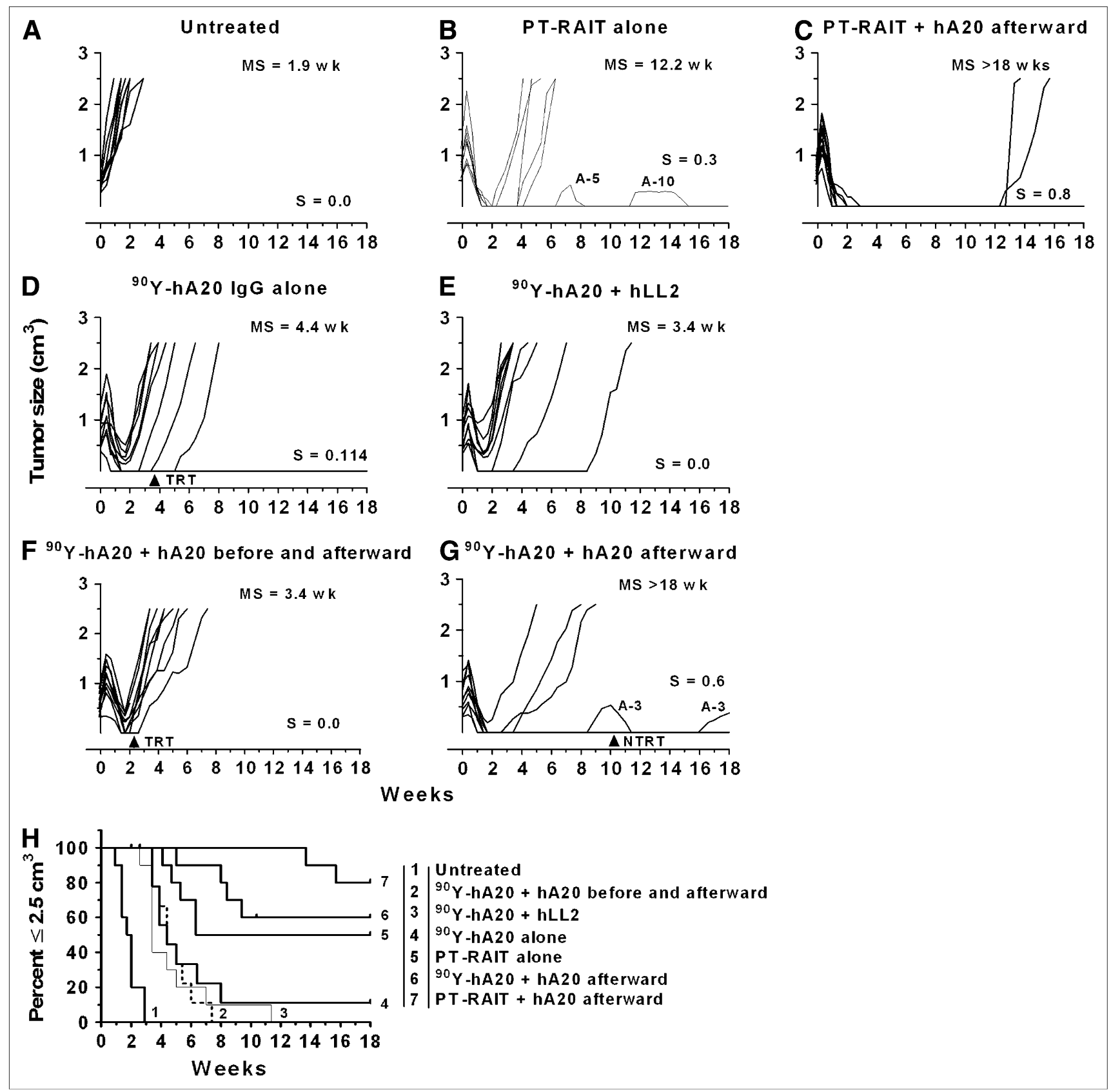

FIGURE 4. Effect of pre- and postdosing with unconjugated veltuzumab on therapeutic activity of ${ }^{90} \mathrm{Y}$-veltuzumab and PTRAIT: Untreated (A), PT-RAIT alone (2.25 nmol/355 $\mu \mathrm{g}$ of TF4, followed $29 \mathrm{~h}$ later with $9.25 \mathrm{MBq} / 0.09 \mathrm{nmol}$ of $90 \mathrm{Y}-\mathrm{IMP}-288)(\mathrm{B})$, PT-RAIT followed $7 \mathrm{~d}$ later with veltuzumab, starting with $1 \mathrm{mg}$ and then with 3 weekly doses of $0.5 \mathrm{mg}$ of veltuzumab (C), 4.81 $\mathrm{MBq}(0.13 \mathrm{mCi} ; 50 \mu \mathrm{g})$ of ${ }^{90} \mathrm{Y}$-veltuzumab alone (D), treatment D coinjected with $1 \mathrm{mg}$ of epratuzumab (hLL2) at $\mathrm{T}_{0}$, followed by weekly injections $\times 3$ of $0.5 \mathrm{mg}$ of epratuzumab (E), $1 \mathrm{mg}$ of veltuzumab administered $1 \mathrm{~d}$ before ${ }^{90}$ Y-veltuzumab (4.81 MBq/50 $\mu \mathrm{g})$ and then followed weekly with $3 \times 0.5 \mathrm{mg}$ of veltuzumab $(F)$, treatment $D$ followed 1 wk later with 1 mg of veltuzumab, with $3 \times 0.5 \mathrm{mg}$ of veltuzumab administered weekly thereafter $(\mathrm{G})$, and percentage of animals remaining with tumors smaller than $2.5 \mathrm{~cm}^{3}(\mathrm{H})$. Ramos tumors were administered ${ }^{90} \mathrm{Y}$-veltuzumab or PT-RAIT with or without unconjugated antibody. For PT-RAIT, $\mathrm{T}_{0}$ is time ${ }^{90}$ Y-IMP-288 was administered. Each growth curve shows fraction of animals cured (S) and median time to $2.5 \mathrm{~cm}^{3}$ (MS). Arrows indicate animals removed from study because of TRT or NTRT event.

mained higher in animals administered the predose $(16.1 \pm$ $2.3 \% \mathrm{ID} / \mathrm{g}$ vs. $22.6 \pm 2.3 \% \mathrm{ID} / \mathrm{g})$. Administration of the predose just $1 \mathrm{~h}$ before ${ }^{111}$ In-veltuzumab reduced tumor uptake the same as when administered $1 \mathrm{~d}$ in advance.
The effect of a 1.0-mg veltuzumab predose on the ${ }^{90} \mathrm{Y}$ veltuzumab treatment without a consolidation course of antibody therapy was examined (Fig. 5). In this situation, more tumors appeared to progress at an earlier time than 


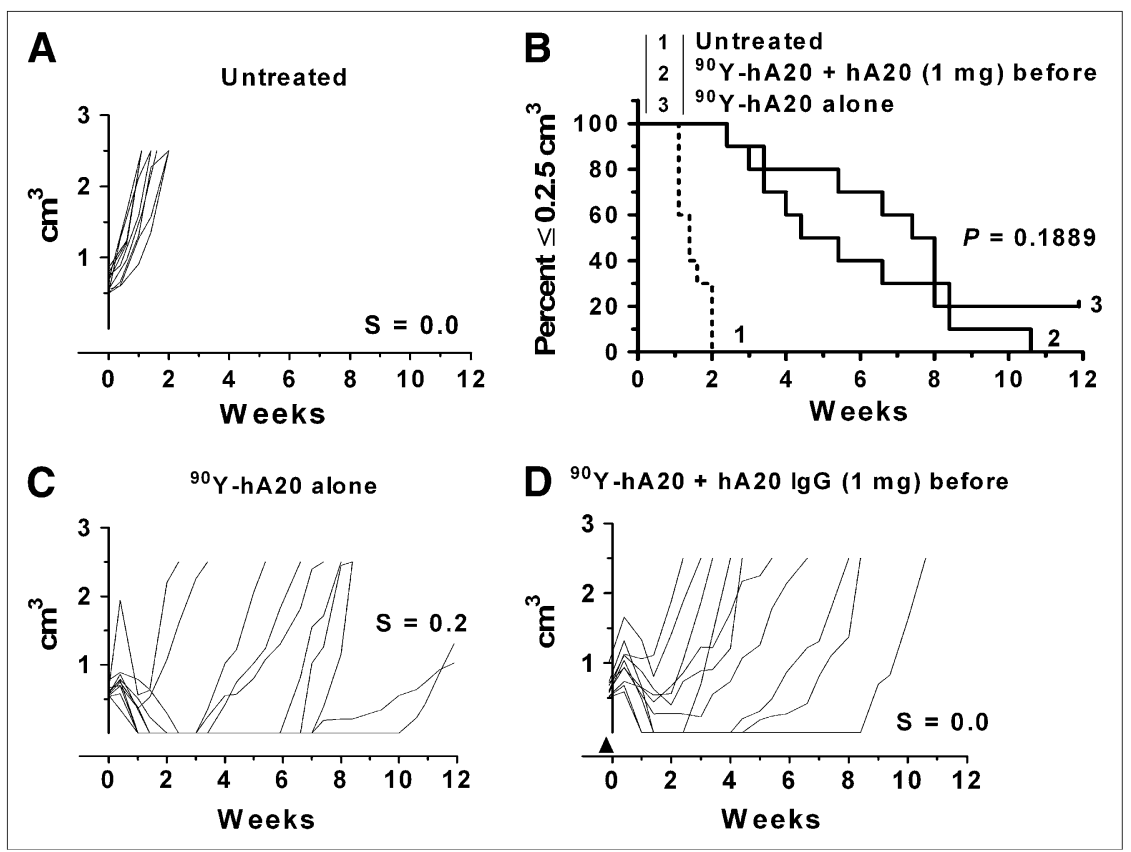

with ${ }^{90}$ Y-veltuzumab administered alone, but although there was a trend to a shorter median survival time in the predosed animals, it was not statistically different (4.9 vs. $7.7 \mathrm{wk}$, respectively; $P=0.1889$ ).

\section{DISCUSSION}

We have been studying a promising new pretargeting approach for treating NHL that is more effective and less toxic than the directly radiolabeled IgG in animal models (9). Because the recombinant humanized anti-CD20 bsMAb (TF4) lacks the Fc-effector portion of an anti-CD20 IgG, we asked whether the fully functional anti-CD20 IgG veltuzumab could be added to PT-RAIT in an effort to derive the same unlabeled-antibody therapeutic benefit afforded with the radioantibody treatments that are administered with nearly $1 \mathrm{~g}$ of unlabeled anti-CD20 IgG. We also examined the addition of anti-CD22 and anti-CD74 antibodies, because these antibodies are active in patients and animals, respectively $(15,22)$. Although lower doses of veltuzumab and milatuzumab alone were active in micrometastatic severe combined immune deficient (SCID) mouse-human B-cell lymphoma models, none of the unconjugated antibodies alone was active against these wellestablished tumors, and the addition of an anti-CD74 or antiCD22 IgG to the PT-RAIT regimen did not improve responses. In contrast, initiation of a veltuzumab treatment $1 \mathrm{wk}$ after PT-RAIT or radioimmunotherapy, compared with radioimmunotherapy alone, significantly enhanced antitumor activity.

This finding led us to examine how a predose of unlabeled veltuzumab might affect PT-RAIT or radioimmunotherapy. Anti-CD20 radioimmunotherapy regimens include a predose of unlabeled anti-CD20 IgG before the radioimmunoconjugate is administered, based on clinical testing that showed uptake was high in the spleen in the absence of a predose. This antigen sink, due to CD20-expressing normal B-cells in the spleen, caused rapid clearance from the blood, particularly in patients with splenomegaly or large tumor burdens. Knox et al. reported a predose of $1 \mathrm{mg} / \mathrm{kg}$ of unlabeled anti-CD20 IgG before ${ }^{90}$ Y-ibritumomab tiuxetan significantly reduced the radiation-absorbed dose to the spleen and significantly increased whole-body (residual) and cardiac blood pool, compared with when no predose was administered (26). Additionally, the percentage of known lesions was visualized better in 4 patients administered a $2.5-\mathrm{kg}$ predose per milligram, compared with separate cohorts administered $1 \mathrm{mg} / \mathrm{kg}$. Radiationabsorbed doses to the tumor were not significantly different between no predose and a $1 \mathrm{mg} / \mathrm{kg}$ predose but were highly variable, possibly masking potential differences. Subsequent studies found no differences in biodistribution when a predose of either 100 or $250 \mathrm{mg}$ of rituximab per square meter was administered before the radioimmunoconjugate, and therefore the higher dose was selected for future treatments because they anticipated that it would provide greater benefit (27). However, because a pretherapy imaging study is performed before the therapy, patients actually receive $500 \mathrm{mg}$ of rituximab per square meter before the ${ }^{90}$ Y-ibritumomab tiuxetan injection. With ${ }^{131} \mathrm{I}$-tositumomab, $450 \mathrm{mg}$ of unlabeled tositumomab are administered before each radioimmunoconjugate injection (before therapy and during therapy). However, clinical studies also indicated that a 95-mg predose of tositumomab slowed the clearance of the radioimmunoconjugate almost as well as did a 450-mg predose (28). Nevertheless, several patients who received a predose of $685 \mathrm{mg}$ of tositumomab before the ${ }^{131}$ I-tositumomab imaging dose experienced tumor shrinkage even before they were administered the ${ }^{131} \mathrm{I}$ tositumomab therapy dose, and these patients were more likely to have a better response after the therapy dose 
$(29,30)$. As the mechanism of action of anti-CD20 antibodies was examined further, the activation of apoptotic signaling of the antibody drew attention to its ability to sensitize cells to radiation $(31,32)$. Thus, it is understandable that these predosing regimens were instituted. Indeed, because clinical studies ultimately confirmed a significantly higher objective response rate with the radioimmunoconjugates than with the unlabeled antibody $(33,34)$, this finding reinforced the belief that these predose amounts were not detrimental to the ability of the radioantibody to provide better therapeutic responses than could unconjugated IgG.

Because antihuman CD20 antibody is not cross-reactive with mouse CD20 (35), mouse models cannot fully mimic the binding competition between a normal B-cell sink and the tumor using antihuman CD20 antibody. However, at the time tositumomab (anti-B1) was being developed, studies in mice also supported a predose to enhance tumor uptake of ${ }^{131} \mathrm{I}$-anti-B1 by reducing splenic uptake and allowing the antibody to clear more slowly from the blood (36). Antibodies from other species and of different murine subclasses do clear differently in mice (37), and thus these results were likely attributed to the IgG2a subclass of antiB1. Although the clearance mechanism is different between mice and humans (i.e., IgG2a rapid clearance in mice is related to Fc-receptor binding, not antigen binding), the results do resemble the conditions seen with anti-CD20 antibodies in patients. Thus, these studies were helpful in identifying the role of predosing when an antigen sink exists; perhaps more important, however, was evidence that excessive predosing (i.e., $1 \mathrm{mg}$ ) reduced tumor uptake (36). The more unusual finding was reported subsequently when the investigators observed that unlabeled anti-B1 antibody elicited a better therapeutic response than did the radioimmunoconjugate in nude mice bearing the Raji Burkitt lymphoma cell line (38). We did not confirm this finding using the humanized anti-CD20 IgG1 in nude mice bearing established subcutaneous Ramos Burkitt lymphoma xenografts, but these results might simply reflect the more active murine IgG2a antibody subclass of the anti-B1 antibody, compared with the human IgG1. Thus, whereas animal models may not reliably predict the therapeutic potential of an unconjugated antibody optimized for human use, when one is active in mice, we can derive an estimate of how the conjugated and unconjugated antibodies contribute to the overall effect, particularly in analyzing dose scheduling.

Although the results showing the benefits of predosing in mice were helpful, it is difficult to reflect accurately the human condition in mice. For example, the initial clinical studies often reported that altered distribution was exacerbated mainly in patients with splenomegaly or large tumor burdens, and thus a model would need to simulate these conditions and define how antigen density within the sink affects targeting. Significant differences in the volume of distribution could also compromise the translation to humans. Antigen content and accessibility in the tumor are other components to consider. Clinical studies did carefully compare no predose to a particular predose level in the same patient, and although some patients were administered minimal and maximal predosing (e.g., 135 and 635 $\mathrm{mg}$ ), the specific biodistribution and targeting data from these patients were not presented in a manner to assess critically the benefit for the larger versus the smaller predose $(28,29,39)$. There was also a lack of substantive data to reflect how tumor localization of the therapeutic product was affected from the cumulative predosing of unlabeled antibody. It may be tempting to speculate that predosing reduced the antigen sink sufficiently to allow better uptake of the therapeutic radioantibody dose, but this speculation ignores the competition for binding sites occurring within the tumor. Ultimately, predosing requirements appear to have been influenced primarily by the imaging or dosimetry findings that suggested that tumor uptake was not compromised by the higher predoses and by the direct observation of improved responses when the higher predoses were administered $(29,30)$ or from the belief that responses would be improved by adding higher amounts of a therapeutically active antibody (27).

Our studies suggest that careful attention needs to be paid to the use of a predose in optimizing the pretargeting of the radiolabeled hapten-peptide in patients, because tumor uptake of the radiolabeled peptide in a pretargeting setting was affected more adversely by the predose than by the direct conjugate. Although we did not directly monitor the distribution of the bsMAb, the data suggest that the predose likely reduces the amount of bsMAb localized to the tumor and, in turn, a lower fraction of the radiolabeled peptide was captured in the tumor. Unlike the direct conjugate, however, the predose did not appreciably affect the tissue uptake of the radiolabeled peptide. Interestingly, in clinical studies using an anti-CD20-streptavidin fusion protein localizing ${ }^{111} \mathrm{In}$-biotin without the benefit of an predose of unlabeled anti-CD20 IgG, whole-body images of patients did not reveal enhanced splenic targeting, and radiation doses to the spleen were more than 6 -fold lower than those to the tumors (40). Thus, predosing requirements for pretargeting procedures might be different from direct radioimmunoconjugates.

Although preclinical and clinical data leave little doubt that in a situation in which a substantial antigen sink exists a predose can favorably alter pharmacokinetics and biodistribution of a radioimmunoconjugate, our studies suggest that the predose should be minimized to reduce competition for binding. This approach should be followed with a more aggressive dosing of the therapeutically active unlabeled antibody. The most robust antitumor response occurred when radioimmunotherapy or PT-RAIT was administered without predosing, followed by unlabeled antibody therapy. Thus, the more radioimmunoconjugate delivered, the more successful the treatment will be.

Unfortunately, animal studies cannot predict the extent to which clinical responses might be affected had the advance dose for the currently approved radioantibody treatments 
been lowered. We focused on the Ramos lymphoma cell line because it has a moderate level of CD20 expression (16), but, more importantly, it grows reliably in BALB/c nude mice without host immunosuppressive conditioning. Although several other cells lines will grow reliably in SCID mice, SCIDs are highly sensitive to radiation, making it difficult to study radioconjugates at effective doses. Thus, although these studies clearly show how unconjugated antiCD20 antibody can be best used in combination with an anti-CD20-targeted radionuclide, the benefit will vary on the basis of several factors in animals and in humans. Ramos in a nude mouse model does show a small percentage of spontaneous regression, thus complicating trial design and extrapolation to clinical studies. Nevertheless, repeated experiments confirmed the basic observations.

Because antibody-dependent cellular toxicity (ADCC) has been considered one of the more important mechanisms of action for anti-CD20 antibodies, the initiation of veltuzumab therapy $1 \mathrm{wk}$ after radioimmunotherapy, at a time when animals had experienced an approximately 80\%-90\% loss in their peripheral blood counts, was still effective (9). One might infer from this result that ADCC is not the principal mechanism of action in this situation, but perhaps the loss in peripheral blood cells does not correlate well with the ability of local effector cells to exert ADCC activity or, alternatively, antibody concentrations were sufficiently persistent in the mouse to engage ADCC during recovery from hematologic toxicity. In this sense, examining the mechanism of action in this setting could be important for determining how to best administer this treatment clinically, particularly because hematologic toxicity has different dynamics in mice and men treated with a radioconjugate.

Finally, we previously found that the same veltuzumab treatment regimen starting $1 \mathrm{~d}$ in advance of ${ }^{90} \mathrm{Y}$-epratuzumab (anti-CD22 IgG) did not alter the biodistribution and tumor uptake of the radioantibody and enhanced antitumor activity of this anti-CD22 radioimmunoconjugate (21). Gopal et al. reported a similar finding, in which the combination of a radiolabeled anti-CD45 antibody with rituximab provided significantly better responses than did the combination with ${ }^{131}$ I-tositumomab anti-CD20, because rituximab blocked the localization of the anti-CD20 radioimmunoconjugate in the tumor by as much as 55\% (20). We observed a similar negative impact on tumor uptake when $1 \mathrm{mg}$ of veltuzumab was administered $1 \mathrm{~d}$, or even $1 \mathrm{~h}$, in advance of ${ }^{111}$ In-veltuzumab. Perhaps because we continued the unlabeled anti-CD20 IgG therapy after ${ }^{90}$ Y-veltuzumab, we did not observe a significant negative impact on the therapeutic activity of ${ }^{90}$ Y-veltuzumab. Nevertheless, combining an active radioantibody with an active unconjugated antibody that binds different antigens (or even noncompeting epitopes) is a promising method.

\section{CONCLUSION}

PT-RAIT using the TF4-trifunctional bsMAb is a promising new therapeutic approach that can be enhanced by combining it with a post-PT-RAIT veltuzumab treatment regimen. Consolidation immunotherapy can provide enhancements similar to directly radiolabeled anti-CD20 IgG. When using a predosing strategy to reduce targeting to a normal tissue sink, the protein dose should be minimized to avoid excessive competition with the binding of the radioimmunoconjugate to the tumor and also to minimize normal-tissue uptake.

\section{ACKNOWLEDGMENTS}

We thank Tom Jackson, Jayson Jebsen, and Omaira Barbarueda at the Garden State Cancer Center, and John Kopinski, Diane Nordstrom, and Meiyu Loo at Immunomedics, Inc., for their excellent technical assistance. This work was supported in part by NIH grant P01-CA103985 from the National Cancer Institute.

\section{REFERENCES}

1. Coiffier B. Rituximab therapy in malignant lymphoma. Oncogene. 2007; 26:3603-3613.

2. Davies AJ. Radioimmunotherapy for B-cell lymphoma: Y90 ibritumomab tiuxetan and I131 tositumomab. Oncogene. 2007;26:3614-3628.

3. Park SI, Press OW. Radioimmunotherapy for treatment of B-cell lymphomas and other hematologic malignancies. Curr Opin Hematol. 2007;14:632-638.

4. Schaefer-Cutillo J, Friedberg JW. Non-myeloablative radioimmunotherapy for non-Hodgkin's lymphoma. Semin Hematol. 2008;45:110-117.

5. Press OW. Evidence mounts for the efficacy of radioimmunotherapy for B-cell lymphomas. J Clin Oncol. 2008;26:5147-5150.

6. Press OW, Corcoran M, Subbiah K, et al. A comparative evaluation of conventional and pretargeted radioimmunotherapy of CD20-expressing lymphoma xenografts. Blood. 2001;98:2535-2543.

7. Sharkey RM, Karacay H, Cardillo TM, et al. Improving the delivery of radionuclides for imaging and therapy of cancer using pretargeting methods. Clin Cancer Res. 2005;11:7109s-7121s.

8. Goldenberg DM, Sharkey RM, Paganelli G, Barbet J, Chatal JF. Antibody pretargeting advances cancer radioimmunodetection and radioimmunotherapy. J Clin Oncol. 2006;24:823-834.

9. Sharkey RM, Karacay H, Litwin S, et al. Improved therapeutic results by pretargeted radioimmunotherapy of non-Hodgkin lymphoma with a new recombinant, trivalent, anti-CD20, bispecific antibody. Cancer Res. 2008;68:5282-5290.

10. Axworthy DB, Reno JM, Hylarides MD, et al. Cure of human carcinoma xenografts by a single dose of pretargeted yttrium- 90 with negligible toxicity. Proc Natl Acad Sci USA. 2000;97:1802-1807.

11. Karacay H, Brard PY, Sharkey RM, et al. Therapeutic advantage of pretargeted radioimmunotherapy using a recombinant bispecific antibody in a human colon cancer xenograft. Clin Cancer Res. 2005;11:7879-7885.

12. Rossi EA, Sharkey RM, McBride W, et al. Development of new multivalentbispecific agents for pretargeting tumor localization and therapy. Clin Cancer Res. 2003;9(suppl):3886S-3896S.

13. Rossi EA, Goldenberg DM, Cardillo TM, McBride WJ, Sharkey RM, Chang CH. Stably tethered multifunctional structures of defined composition made by the dock and lock method for use in cancer targeting. Proc Natl Acad Sci USA. 2006;103:6841-6846.

14. Brard PY, Karacay H, Stein R, et al. A divalent hapten-peptide induces apoptosis in human non-Hodgkin lymphoma cell lines targeted by anti-CD20xanti-hapten bispecific antibodies. Clin Cancer Res. 2007;13(18 suppl):5564S-5571S.

15. Stein R, Qu Z, Cardillo TM, et al. Antiproliferative activity of a humanized antiCD74 monoclonal antibody, hLL1, on B-cell malignancies. Blood. 2004;104: 3705-3711.

16. Stein R, Qu Z, Chen S, et al. Characterization of a new humanized anti-CD20 monoclonal antibody, IMMU-106, and its use in combination with the humanized anti-CD22 antibody, epratuzumab, for the therapy of non-Hodgkin's lymphoma. Clin Cancer Res. 2004;10:2868-2878.

17. Carnahan J, Stein R, Qu Z, et al. Epratuzumab, a CD22-targeting recombinant humanized antibody with a different mode of action from rituximab. Mol Immunol. 2007;44:1331-1341. 
18. Goldenberg DM, Rossi EA, Stein R, et al. Properties and structure-function relationships of veltuzumab (hA20), a humanized anti-CD20 monoclonal antibody. Blood. 2009;113:1062-1070.

19. DeNardo GL, Tobin E, Chan K, Bradt BM, DeNardo SJ. Direct antilymphoma effects on human lymphoma cells of monotherapy and combination therapy with CD20 and HLA-DR antibodies and ${ }^{90}$ Y-labeled HLA-DR antibodies. Clin Cancer Res. 2005;11(19 suppl):7075S-7079S.

20. Gopal AK, Press OW, Wilbur SM, Maloney DG, Pagel JM. Rituximab blocks binding of radiolabeled anti-CD20 antibodies (Ab) but no radiolabeled antiCD45-Ab. Blood. 2008;112:830-835.

21. Mattes MJ, Sharkey RM, Karacay H, Czuczman MS, Goldenberg DM. Therapy of advanced B-lymphoma xenografts with a combination of ${ }^{90} \mathrm{Y}$-anti-CD22 IgG (epratuzumab) and unlabeled anti-CD20 IgG (veltuzumab). Clin Cancer Res. 2008;14:6154-6160.

22. Leonard JP, Goldenberg DM. Preclinical and clinical evaluation of epratuzumab (anti-CD22 IgG) in B-cell malignancies. Oncogene. 2007;26:3704-3713.

23. Morschhauser F, Leonard JP, Fayad L, et al. Humanized anti-CD20 antibody, veltuzumab, in refractory/recurrent non-Hodgkin's lymphoma: phase I/II results. $J$ Clin Oncol. In press.

24. Griffiths GL, Govindan SV, Sharkey RM, Fisher DR, Goldenberg DM. ${ }^{90}$ Y-DOTA-hLL2: an agent for radioimmunotherapy of non-Hodgkin's lymphoma. J Nucl Med. 2003;44:77-84.

25. Food and Drug Administration, Center for Drug Evaluation and Research (CDER). Guidance for Industry: Estimating the Maximum Safe Starting Dose in Initial Clinical Trials for Therapeutics in Adult Healthy Volunteers. Rockville, MD: Food and Drug Administration, Center for Drug Evaluation and Research, July 2005. Available at: http://www.fda.gov/Cder/Guidance/5541fnl.htm. Accessed January 8, 2009.

26. Knox SJ, Goris ML, Trisler K, et al. Yttrium-90-labeled anti-CD20 monoclonal antibody therapy of recurrent B-cell lymphoma. Clin Cancer Res. 1996;2:457-470.

27. Witzig TE, White CA, Wiseman GA, et al. Phase I/II trial of IDEC-Y2B8 radioimmunotherapy for treatment of relapsed or refractory $\mathrm{CD} 20(+) \mathrm{B}$-cell non-Hodgkin's lymphoma. J Clin Oncol. 1999;17:3793-3803.

28. Wahl RL, Zasadny KR, MacFarlane D, et al. Iodine-131 anti-B1 antibody for B-cell lymphoma: an update on the Michigan Phase I experience. J Nucl Med. 1998;39:21S-27S.
29. Kaminski MS, Zasadny KR, Francis IR, et al. Iodine-131-anti-B1 radioimmunotherapy for B-cell lymphoma. J Clin Oncol. 1996;14:1974-1981.

30. Kaminski MS, Zasadny KR, Francis IR, et al. Radioimmunotherapy of B-cell lymphoma with $\left[{ }^{131} \mathrm{I}\right]$ anti-B1 (anti-CD20) antibody. $N$ Engl J Med. 1993; 329:459-465.

31. Skvortsova I, Skvortsov S, Popper BA, et al. Rituximab enhances radiationtriggered apoptosis in non-Hodgkin's lymphoma cells via caspase-dependent and -independent mechanisms. J Radiat Res (Tokyo). 2006;47:183-196.

32. Kapadia NS, Engles JM, Wahl RL. In vitro evaluation of radioprotective and radiosensitizing effects of rituximab. J Nucl Med. 2008;49:674-678.

33. Davis TA, Kaminski MS, Leonard JP, et al. The radioisotope contributes significantly to the activity of radioimmunotherapy. Clin Cancer Res. 2004;10:7792-7798.

34. Witzig TE, Gordon LI, Cabanillas F, et al. Randomized controlled trial of yttrium-90-labeled ibritumomab tiuxetan radioimmunotherapy versus rituximab immunotherapy for patients with relapsed or refractory low-grade, follicular, or transformed B-cell non-Hodgkin's lymphoma. J Clin Oncol. 2002;20:24532463.

35. Uchida J, Lee Y, Hasegawa M, et al. Mouse CD20 expression and function. Int Immunol. 2004;16:119-129.

36. Buchsbaum DJ, Wahl RL, Glenn SD, Normolle DP, Kaminski MS. Improved delivery of radiolabeled anti-B1 monoclonal antibody to Raji lymphoma xenografts by predosing with unlabeled anti-B1 monoclonal antibody. Cancer Res. 1992;52:637-642.

37. Sharkey RM, Natale A, Goldenberg DM, Mattes MJ. Rapid blood clearance of immunoglobulin G2a and immunoglobulin G2b in nude mice. Cancer Res. 1991;51:3102-3107.

38. Buchsbaum DJ, Wahl RL, Normolle DP, Kaminski MS. Therapy with unlabeled and ${ }^{131} \mathrm{I}$-labeled pan-B-cell monoclonal antibodies in nude mice bearing Raji Burkitt's lymphoma xenografts. Cancer Res. 1992;52:6476-6481.

39. Wahl RL. Tositumomab and ${ }^{131}$ I therapy in non-Hodgkin's lymphoma. $\mathrm{J} \mathrm{Nucl}$ Med. 2005;46(suppl 1):128S-140S.

40. Forero A, Weiden PL, Vose JM, et al. Phase 1 trial of a novel anti-CD20 fusion protein in pretargeted radioimmunotherapy for B-cell non-Hodgkin lymphoma. Blood. 2004;104:227-236. 